\title{
Wo liegt die vordere Grenze des ophthalmoskopisch sichtbaren Augenhinter- grundes?
}

\author{
Von \\ Dr. A. Groenouw, \\ Assistenzarzt an der Universitäts-Klinik für Augenkranke \\ zu Breslau.
}

Mit 2 Figuren im Text.

Veranlasst durch Herrn Geheimrath Förster bei Gelegenheit einer Cysticercus-Extraction trat ich der Frage näher, wie weit man im Stande sei, mit dem Augenspiegel die Netzhaut nach vorn hin zu überblicken. Die genaue Kenntniss dieser Thatsache erscheint ausserordentlich wichtig für die Entfernung eines unter der Netzhaut oder im Glaskörper gelegenen Fremdkörpers z. B. einer Cysticercusblase oder eines Eisensplitters, da auf diese Weise ein gutes Mittel zur genauen Ortsbestimmung des Fremdkörpers und somit zur richtigen Schnittführung behufs Extraction desselben gegeben ist.

Die angeregte Frage ist merkwürdiger Weise bisher noch so gut wie gar nicht ventilirt worden, trotzdem über die Theorie der Augenspiegeluntersuchung und die Grösse des ophthalmoskopischen Bildes schon eine umfangreiche Literatur existirt. Die einzige Notiz über diesen Punkt 
stammt von Douders ${ }^{1}$, welcher an zwei emmetropischen Augen fand, dass die vordere Grenze des sichtbaren Augenhintergrundes im horizontalen Meridian innen und aussen sich gleichweit erstreckt, nämlich je $8 \mathrm{~mm}$ nach hinten von dem Hornhautrande liegt. Donders hat diese Frage aber nur flüchtig gestreift, da er bei seiner Arbeit einen ganz anderen $Z$ weck verfolgte.

Suchen wir uns zunächst klar zu machen, worauf es beruht, dass wir die vorderste Parthie der Netzhaut mit dem Augenspiegel nicht sehen können, so werden wir dies am einfachsten erreichen, wenn wir den Gang desjenigen Strahles betrachten, welcher von dem vordersten eben noch sichtbaren Punkte des Augengrundes herkommt. Diesen Strahl wollen wir als "Grenzstrahl" bezeichnen. Er hat die Eigenschaft, von einem Punkte der Netzhaut auszugehen, von welchem eben nur dieser einzige Strahl durch Linse, Pupille und Cornea aus dem Auge herausgelangen kann, während von jedem mehr nach hinten gelegenen Netzhautpunkte mehr als ein Strahl, von jedem weiter vorn liegenden überhaupt kein Strahl mehr aus dem Auge heraustreten kann.

Es wird offenbar ganz gleichgültig sein, ob wir untersuchen, wo derjenige Punkt der Netzhaut liegt, von welchem eben noch ein Strahl aus dem beobachteten Auge herausgelangen kann, oder welchen Punkt der Netzhaut derjenige Strahl trifft, welcher gerade noch in das beobachtete Auge hineintreten kann. Mit anderen Worten ausgedrückt wird die Frage: "Welches ist der vorderste Punkt der Netzhaut, welchen man noch sehen kann?" mit der Frage zusammenfallen: "Welches ist der peripherste Netzhautpunkt, zu welchem vop aussen noch Licht hingelangen kann?"

Tritt nämlich auf einem bestimmten Wege ein Licht-

1) Archiv für Ophthalmologie, XXIII, 2, pag. 264. 
Wo liegt die vordere Grenze des etc. Augenhintergrundes? 31 strahl in das Auge hinein, so muss auch umgekehrt auf demselben Wege ein Strahl aus dem Auge heraustreten können. Dieser Satz widerspricht dem Gesetze von der totalen Reflexion durchaus nicht, da es eben unmöglich ist, dass ein aus einem optisch dünneren in ein dichteres Medium eintretenden Strahl in letzterem einen so grossen Brechungswinkel bildet, dass er bei umgekehrtem Verlaufe total reflectirt werden müsste.

Wir betrachten also, welches der äusserste Strahl ist, welcher von aussen noch zur Netzhaut gelangen kann, da diese Betrachtungsweise bedeutend einfacher ist, als die umgekehrte. Tritt ein Strahl in der Richtung der optischen Achse ins Auge hinein, so trifft er den hinteren Pol der Retina, bildet seine Richtung einen Winkel mit der optischen Achse, so gelangt er zu irgend einem anderen, mehr nach dem Ciliarkörper hin gelegenen Punkte des Augengrundes. Dieser Punkt wird um so weiter nach vorn hin rücken, je grösser der Winkel des einfallenden Strahles mit der optischen Achse ist. Doch ist dem Anwachsen dieses Winkels eine bestimmte Grenze gesetzt, da sonst der eintretende Strahl nicht mehr durch die Pupille zur Retina gelangt, sondern von der Iris aufgefangen wird, ohne den Augengrund zu erreichen. Wann tritt dieser Moment ein?

Fig. 1 zeigt einen Horizontalschnitt durch den Bulbus in der optischen Achse ABM. M ist der Mittelpunkt des grössten Kreises $A N R L R_{3} N_{1}$, in welchem die Sclera von dem Schnitte getroffen wird. $\mathrm{NAN}_{1}$ ist die durchsichtige Cornea, $\mathrm{NBN}_{1}$ ihre Basis, welche auf der optischen Achse AM senkrecht steht. $\mathrm{N}_{1} \mathrm{M}_{3}$ ist der Krümmungsradius am Hornhautrande, der Mittelpunkt dieser Krümmung braucht jedoch nicht gerade auf der optischen Achse zu liegen.

$R_{1} G E R_{2}$ ist die vordere Linsenfläche, deren Krümmungsmittelpunkt in $M_{1}$ liegt, während die Lage des Krümmungsmittelpunktes der hinteren Linsenfläche durch $\mathrm{M}_{2}$ gegeben ist. 
$\mathrm{RR}_{1} \mathrm{R}_{2} \mathrm{R}_{3}$ stellt die Iris dar. $\left.{ }^{1}\right)$

Denken wir uns nun zunächst einmal alle durchsichtigen Theile aus dem Auge entfernt, so dass nur das undurchsichtige Gerüst zurückbleibt. Wir würden dies erreichen, wenn wir die Hornhaut an ihrer Basis $\mathrm{NBN}_{1}$ durch einen Verticalschnitt abtrügen und Linse nebst Glaskörper

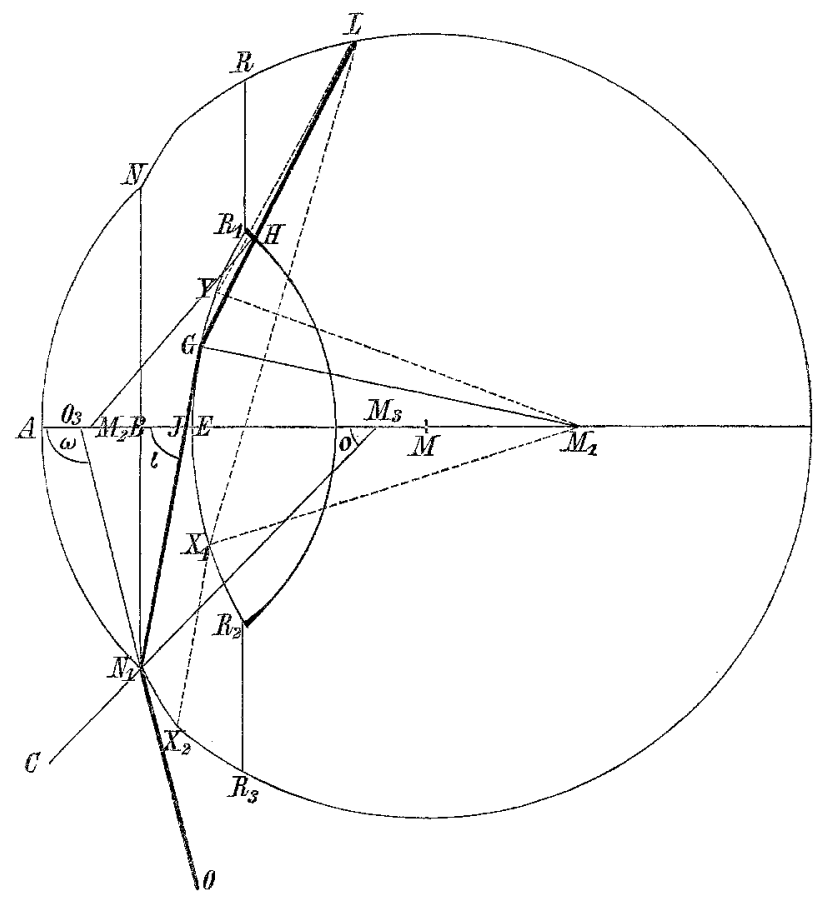

Fig. 1.

aus dem Inneren des Bulbus entfernten; so dass der von der Sclera umschlossene Hohlraum von Luft erfüllt würde,

1) In Fig. 1 ist zwar der Verlauf des Strahles in Cornea und Linse in vierfacher Vergrösserung genau wiedergegeben, doch ist die Krümmung der Sclera zu klein und die Iris zu weit nach hinten verschoben gezeichnet worden. 
Wo liegt die vordere Grenze des etc. Augenhintergrundes? 33

wobei wir natürlich voraussetzen, dass Iris und Sclera ihre normale Lage und Krümmung beibehalten. Dann ragt die undurchsichtige Sclera von $N_{1}$ bis $R_{3}$ resp. von $N$ bis $R$ als Ring von etwa $1 \mathrm{~mm}$ Breite über die Iris hervor, und wir werden den Augengrund nur bis zu einem Punkte hin überblicken können, in welchem ihn eine Linie $N_{1} R_{1}$, d.h. eine Verbindungslinie des Hormhautrandes mit dem gegenüberliegenden Pupillarrande trifft, da wir eben nur in einer geraden Linie, aber nicht um eine Ecke herum sehen können. Dieser Punkt liegt etwa $6 \mathrm{~mm}$ hinter dem Hornhautfalze. Wir werden also selbst unter diesen günstigsten Umständen einen nicht ganz $6 \mathrm{~mm}$ breiten unmittelbar hinter dem limbus corneae gelegenen Streifen des Augengrundes ophthalmoskopisch nicht wahrnehmen können.

Sobald wir uns aber die brechenden Medien des Auges wieder an ihre normale Stelle gesetzt denken, ändern sich die Verhältnisse.

Sei $O N_{1}$ ein Strahl, welcher am Hornhautrande also durch den periphersten Theil der durchsichtigen Cornea ins Auge eintritt, so wird dieser Strahl durch die brechenden $\mathrm{Me}-$ dien des Auges von seiner Bahn abgelenkt werden, zunächst in der Richtung $N_{1} G$ verlaufen, darauf durch die vordere und hintere Linsenfläche noch zweimal gebrochen werden und in der Richtung GHL schliesslich zu dem Punkte $\mathrm{I}_{\text {. }}$ gelangen. Dieser Punkt $\mathrm{L}$ wird dem Hornhautfalze um so näher liegen, d. b. der in $O$ befindliche Beobachter wird einen um so mehr nach vorn gelegenen Punkt des Augengrundes erblicken, je grösser der $\Varangle \mathrm{N}_{1} \mathrm{O}_{3} \mathrm{~A}==\infty$ ist, welchen die Verlängerung des Strahles $O N_{1}$ mit der optischen Achse $\mathrm{AO}_{3} \mathrm{~B}$ bildet.

Dieser Winkel darf aber eine bestimmte Grenze nicht überschreiten, da sonst der Strahl $O N_{1}$, oder richtiger seine durch die Hornhaut gebrochene Fortsetzung $N_{1} G$, gar nicht mehr durch die Pupille $\mathrm{R}_{1} \mathrm{R}_{2}$ zur Retina gelangt, sondern vor der Pupille vorbeigeht, um auf die Iris $\mathrm{RR}_{1}$, auf die

v. Graefe's Archir fur Ophthalmologie, XXXV. 3. 
Sclera RN oder gar auf die entgegengesetzte Hornhauthaalfte $\mathrm{AN} z u$ treffen.

Bei $\mathrm{N}_{1}$ wird der Strabl stets unter einem so grossen Winkel in die Hornhaut eintreten müssen, dass er nach seiner Brechung noch in der Richtung $N_{1} G$ in die Pupille hineingelangen kann. Dies ist aber immer möglich, da einerseits aus einem dünneren Medium (Luft) in ein dichteres (Hornhaut-Kammerwasser) ein Strahl unter jedem beliebigen Winkel eintreten kann, andererseits der Grösse des Einfallswinkels in $N_{1}$ nur durch die sehr weit zurücktretende undurchsichtige Bulbuswand $\mathrm{N}_{1} \mathrm{R}_{3}$ eine Grenze gesteckt ist.

Der gebrochene Strahl muss nun, falls er der Grenzstrahl ist, innerhalb der vorderen Kammer so verlaufen, dass er mit der optischen Achse wiederum einen möglichst. grossen Winkel $\mathrm{N}_{1} \mathrm{JA}=\iota$ bildet, ohne jedoch schon durch das Diaphragma $R_{1}$ abgefangen zu werden. Man wird zunächst unwillkürlich annehmen, der Strahl müsse dann in der Richtung $\mathrm{N}_{1} \mathrm{R}_{1}$, also in einer Verbindungslinie des Hornhautrandes mit dem gegenïberliegenden Pupillarrande verlaufen. Doch ist dies gar nicht möglich, da die Linse etwas über die Pupillarebene hervorragt und also ein Strahl, welcher in der Richtung $N_{1} R_{1}$ verläuft, ehe er noch in $R_{1}$ angelangt, schon auf die Linse trifft und durch sie gebrochen wird. Der Grenzstrahl wird vielmehr in der von $N_{1}$ an die vordere Linsenfläche gelegten Tangente $N_{1} G$ verlaufen, da dies derjenige von $N_{1}$ ausgehende Strahl ist, welcher den grössten Winkel mit der optischen Achse bildet und doch noch die Linse trifft. Der $\Varangle \mathrm{N}_{1} \mathrm{GM}_{1}$, welcher gleich dem Einfallswinkel des Strahles $N_{1} G$ gegen die vordere Linsenfläche ist, beträgt also $1 \mathrm{R}$ und der zugehörige Brechungswinkel $\mathrm{M}_{1} \mathrm{GL}$ ist der grösste Winkel, unter welchem ein Strahl gegen das Einfallsloth $\mathrm{GM}_{1}$ geneigt sein darf, um aus der linse ins Kammerwasser austreten zu können, ohne total reflectirt zu werden. 
Wo liegt die rordere Grenze des etc. Augenhintergrundes? 35

Der Strahl GL wird in H beim Austritt aus der hinteren Linsenfläche in den Glaskörper noch einmal eine Brechung von dem Einfallslothe $\mathrm{M}_{2} \mathrm{H}$ hinweg erleiden, doch ist diese Brechung, da der $\Varangle \mathrm{M}_{2} \mathrm{HG}$ stets nur klein ist, so gering, dass wir sie vernachlässigen können.

Da der Grenzstrahl $\mathrm{N}_{1} \mathrm{G}$ die Tangente an die vordere Linsenfläche bildet, so wird er solange von dem Pupillarrande der Iris nicht erreicht werden, als dieser sich weiter peripher befindet als der Punkt G. Sobald also die halbe Pupillenweite grösser ist als EG, wird das Stück des Augengrundes, welches wir ophthalmoskopisch überblicken, durch Erweiterung der Pupille nicht vergrössert werden. Da nun EG einem Pupillendurchmesser (nicht Radius) von etwa $3,5 \mathrm{~mm}$ entspricht, so ist die vordere Grenze des sichtbaren Augengrundes verhältnissmässig wenig von der Pupillenweite abhängig. Wenn wir trotzdem bei erweiterter Pupille den Augengrund weiter iibersehen, als bei enger, so beruht dies rielmehr auf dem durch die Weite der Pupille vermehrten lichteinfall, als darauf, dass bei enger $\mathrm{Pu}-$ pille die Iris die Strahlen, welche von dem periphersten Theile des Augengrundes herkommen, nicht in unser Auge gelangen lässt.

Die Grösse des sichtbaren Theiles der Netzhaut hängt also von der Hornhaut und ihrer Krümmung gar nicht ab, von der Weite der Pupille nur insoweit, als letztere nicht gar zu eng sein darf, dagegen fast ausschliesslich von der Lage der vorderen Linsenfläche zum Hornhautrande, so dass wir um so weiter nach vorn hin blicken können, je näher die Linse dem. Hornhautrande liegt, je enger also die vordere Kammer ist. Wir müssten also bei Accommodation des untersuchten Auges, wobei sich die Linse in die vordere Kammer hinein stärker vorwölbt, ihr Brechungsindex aber gleich bleibt, ein grösseres Stück des Augengrundes übersehen, wenn hierbei nicht der durch die Enge der Pupille verminderte Lichteinfall hinderlich wäre. 
Bisher baben wir der Einfachheit halber den Weg betrachtet, auf welchem ein ron aussen in das Auge eintretender Strahl zu einem möglichst weit nach vorn gelegenen Punkte des Augengrundes gelangen muss, wobei wir Fon der Voraussetzung ausgingen, dass dieser Punkt auch der peripherste Netzhautpunkt ist, von welchem umgekehrt noch Licht aus dem Auge herausgelangen kann, doch kann vielleicht noch ein Beweis dafür verlangt werden.

Nehmen wir irgend einen von dem Punlte $L$ ausgebenden Strahl z. B. LY, welcher die Linse näher ihrem Rande trifft als der Strahl $L G$, in die Linse hineintritt und in $\mathrm{Y}$ zux vorderen Linsenfläche gelangt, so wird derselbe mit dem Radius $\mathrm{YM}_{1}$ dieser Fläche, welcher zugleich das Einfallsloth ist, den $\Varangle \mathrm{LYM}_{1}$ bilden. Dieser Winkel ist aber grösser als $\Varangle L G M_{1}$, wie sich leicht geometrisch zeigen lässt, wenn man bedenkt, dass $\mathrm{L}$ ein Punkt ausserhalb des Kreises $M_{1}$ ist, and $L$ und $M_{1}$ mit einem Punkte der Peripherie dieses Kreises verbunden werden, wobei der von ihnen eingeschlossene Winkel um so grösser ist, je näher $\mathrm{Y}$ an $\mathrm{L}$ heranruickt.

$\Varangle \mathrm{LGM} \mathrm{M}_{1}$ stellt den grössten Winkel dar, unter welchem ein Strahl noch aus der Linse ins Kammerwasser heraustreten kann, wird der Einfallswinkel noch grösser, z. B. $=\mathrm{LYM}_{1}$, so kann der Strahl LY nicht mehr aus der Linsensubstanz heraustreten, sondern wird in $\mathrm{X}$ total reflectirt, gelangt so zu irgend einem anderen Punkte der vorderen Linsenfläche, in welchem er nochmals reflectirt wird, und kann so schliesslich wieder in den Glaskörper zurückgelangen, jedenfalls aber nicht aus der Linse ins Kammerwasser austreten.

Denken wir uns nun, es ginge von L ein anderer Strahl aus, welcher durch Glaskörper und Linse zur vorderen Fläche der letzteren gelangt, und dieselbe nicht in $G$, sondern in einem weiter nach $R_{2}$ zu gelegenen Punkte z. B. in $X_{1}$ trifft, so wird dieser Strahl $L X_{1}$ mit dem Einfallslothe $\mathrm{X}_{1} \mathrm{M}_{1}$ 
Wo liegt die vordere Grenze des etc. Augenhintergrundes? 37 einen kleineren Winkel als $\mathrm{LGM}_{1}$ bilden, er wird also nicht total reflectirt werden, sondern aus der Linse heraustreten und in der Richtung $\mathrm{X}_{1} \mathrm{X}_{2}$ verlaufen. Aber auch dieser Strahl wird nicht in das Auge des Beobachters gelangen, vielmehr auf den undurchsichtigen Theil der Hormhaut resp. Sclera zwischen $N_{1}$ und $R_{3}$ treffen.

Von $L$ aus kann also nur ein einziger Strahl $L G$ durch die Linse ins Kammerwasser heraustreten, jeder andere Strahl dagegen wird entweder, wenn er einen Punkt der vorderen Linsenfläche trifft, welcher von $G$ aus weiter nach $R_{1}$ hin liegt, total reflectirt werden, oder aber, wenn er auf das Stiick $\mathrm{GR}_{2}$ der vorderen Linsenfläche trifft, so wird er zwar aus der Linse heraustreten können, jedoch von dem undurchsichtigen Theile der Bulbuswand $N_{1} R_{3}$ aufgefangen werden. Die Brechung an der hinteren Linsenfläche haben wir hierbei aus den oben angeführten Gründen vernachlässigt.

Nehmen wir statt $L$ einen Punkt, welcher noch weiter vorn liegt zwischen $L$ und $R$, so wird der Winkel, welchen ein von ihm ausgehender Strahl an der vorderen Linsenfläche mit deren Radius bildet, stets grösser sein als der Winkel, welchen ein von $L$ nach demselben Punkte der Linsenvorderfläche gehender Strahl mit demselben Radius bildet, es wird also ein solcher Strahl ebenfalls entweder total reflectirt werden oder auf das undurchsichtige Stück $\mathrm{N}_{1} \mathrm{R}_{3}$ der Bulbuswand treffen.

Der Punkt L ist also wirklich der peripherste Punkt des Augenhintergrundes, von welchem aus noch Licht aus dem Auge heraus gelangen kann, d. h. welcher mit dem Augenspiegel noch sichtbar ist.

Auf Grund dieser theoretischen Betrachtungen ist es möglich, aus dem Neigungswinkel des Strahles $0 N_{1}$ gegen die optische Achse $\left(\Varangle \omega=\mathrm{AO}_{3} \mathrm{~N}_{1}\right.$ ) und den sonstigen Krümmungs- und Brechungsverhältnissen des Auges die 
Entfernung des Punktes $L$ von dem Hornhautrande $N$ zu berechnen.

Am einfachsten erscheint es, den $\Varangle o$ all den Knotenpunkt anzutragen und so die Grösse NL zu bestimmen. Indessen ergiebt diese Methode stets für NL eine etwas zu geringe Länge gegenüber der auf die später zu erwähnende Weise direct gemessenen Entfernung der beiden Punkte von einander, da der Knotenpunkt des schematischen Auges wahrscheinlich etwas zu weit nach vorn liegt, ein Resultat zu dem auch Donders gekommen ist. Die Differenz ist allerdings nicht sehr ins Gewicht fallend und nur wenig grösser als bei der noch zu besprechenden Methode, doch hat dieses Verfahren ein grosses theoretisches Bedenken. Bei allen Betrachtungen über die Brechung durch Linsen resp. gekrümmte Flächen gehen wir nämlich von der Voraussetzung aus, dass die einfallenden Strahlen mit der optischen Achse einen kleinen Winkel bilden. In dem vorliegenden Falle ist der fragliche Winkel aber sehr gross, so dass man mit Recht erhebliche Bedenken gegen die Gültigkeit der allgemeinen Formeln für Bild- und Objectweite und gegen die Einführung eines Knotenpunktes für mehrere brechende Flächen erheben kamn.

Um diesem Einwande zu entgehen, wollen wir die Strecke NL zu berechnen suchen, indem wir den Gang des Grenzstrahles in der oben erörterten Weise der Berechnung zu Grunde legen.

In Fig. $2^{1}$ ) ist ebenso wie in Fig. $1 \mathrm{NN}_{1}$ die Hornhautbasis, AM die optische Achse, M der Mittelpunkt der Kugeloberfläche der Selera, auf welcher $\mathrm{L}$ liegt, $\mathrm{M}_{\mathbf{1}}$ der Krümmungsmittelpunkt der vorderen Linsenfläche EG.

Ist $0 \mathrm{~N}_{1}$ der Grenzstrahl, welcher mit der optischen Achse den $\Varangle \mathrm{N}_{1} \mathrm{O}_{3} \mathrm{~A}=\omega$ bildet und ist $\mathrm{N}_{1} \mathrm{M}_{3}$ der Krüm-

1) Fig. 2 ist schematisch, die Lage und Grössenverhältnisse der Theile zu einander sind etwas geändert. 
Wo liegt die vordere Grenze des etc. Augenhintergrundes? 39 mungsradius am Hornhautrande, dessen Neigungswinkel gegen die optische Achse $\Varangle \mathrm{AM}_{3} \mathrm{~N}_{1}=0$ ist, so ist $\Varangle 0 \mathrm{~N}_{1} \mathrm{C}$ $=\alpha_{1}$, der Einfallswinkel des Strahles $\mathrm{N}_{1} \mathrm{O}$ gegen die Hornhaut. Verläuft dieser Strahl nach der Brechung in der Richtung $\mathrm{N}_{1} \mathrm{G}$, so ist $\Varangle G \mathrm{~N}_{1} \mathrm{M}_{3}=\beta_{1}$ der Brechungswinkel

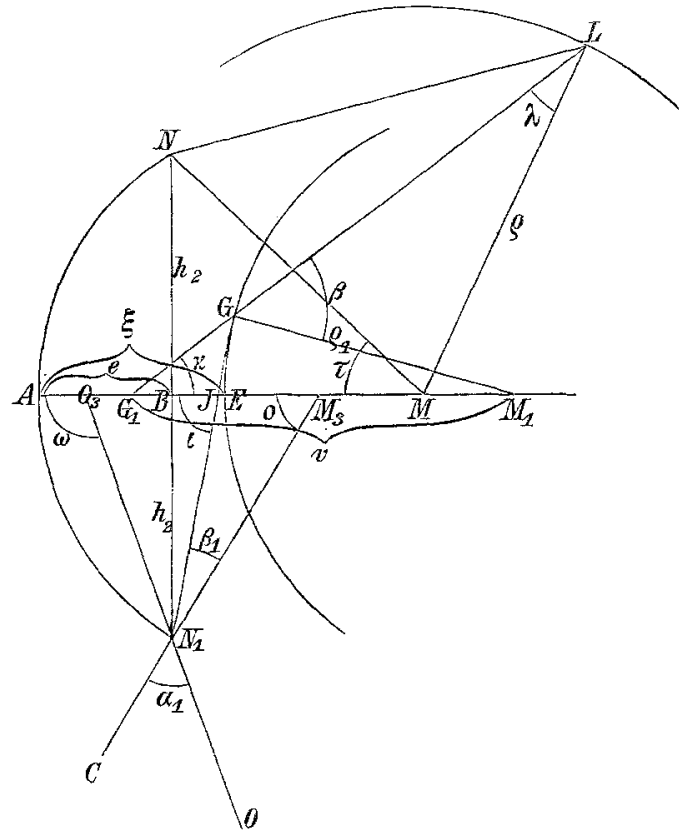

Fig. 2.

und nach dem allgemeinen Brechungsgesetze besteht die Gleichung

$$
\sin \beta_{1}=\frac{1}{n_{0}} \sin \alpha_{1}
$$

wenn wir unter $n_{0}$ den Brechungsindex von Hornhaut, Kammerwasser und Glaskörper (nach Matthiessen =1,3350) verstehen.

$$
\Varangle \alpha_{1}=\Varangle \omega-o \quad \text { daher }
$$

1) $\quad \sin \beta_{1}=\frac{1}{n_{0}} \sin (\omega-o)$. 
Der in $N_{1}$ gebrochene Strahl $N_{1} G$ bildet die Tangente an die vordere Linsenfläche (daher $\Varangle \mathrm{N}_{1} \mathrm{GM}_{1}=1 \mathrm{R}$ ) bier wird er nochmals gebrochen und verläuft in der Richtung GL.

Der Brechungswinkel $M_{1} G L$ sei $\beta$, so wird dessen Grösse bestimmt durch die Gleichung

$$
\sin \beta=\frac{n_{0}}{n_{1}}
$$

wenn wir mit $n_{1}$ den Brechungsindex der äussersten Corticalschichten der Linse bezeichnen (nach Matthiessen $=1,3750$ ).

Nur die äussersten Corticalschichten kommen hier in Betracht, da der gebrochene Strahl, wie man sich an einer genau construirten Zeichnung z. B. Fig. 1 leicht uiberzeugen kann, in den oberflächlichsten Parthieen der Linse verläuft, ohne die tieferen stärker brechenden Schichten zu berühren, ein Umstand, der die Berechnung ausserordentlich vereinfacht, indem wir auf die Linse als einen geschichteten Körper keine Rücksicht zu nehmen brauchen, sondern sie für die hier eine Rolle spielende Zone als homogen betrachten können.

Bei den angegebenen Brechungsexponenten ist

$$
\Varangle \beta=76^{\circ} 36^{\prime} 22^{\prime \prime} \text {. }
$$

Um nun das Stück NL zu berechnen, verlängern wir zunächst $L G$ bis $G_{1}$ und führen die aus Fig. 2 ersichtlichen Bezeichnungen ein.

Aus $\triangle G_{1} G M_{1}$ ergiebt sich

ferner aus $\triangle J M_{1} G$

$$
\Varangle x=\Varangle \beta-\not \mathrm{G}_{1} \mathrm{M}_{1} \mathrm{G}
$$

und aus $\triangle J_{M_{3}} N_{1}$

$$
\Varangle \mathrm{G}_{1} \mathrm{M}_{1} \mathrm{G}=1 \mathrm{R}-\iota
$$

$$
\chi \iota=o+\beta_{1}
$$

woraus durch Eintragen dieser Werthe in die erste Gleichung schliesslich folgt

3) $\quad \chi x=\beta+\beta_{1}+o-\mathrm{R}$. 
Wo liegt die vordere Grenze des etc. Augenhintergrundes? 41

Bezeichnen wir $G_{1} \mathbf{M}_{1}$ mit $\nabla, G_{1}$ als Radius der vorderen Linsenfläche mit $\varrho_{1}$, so folgt aus $\triangle G_{1} M_{1} G$

daher ist

$$
\mathrm{G}_{1} \mathrm{M}_{1}: \mathrm{GM}_{1}=\sin \beta: \sin \varkappa
$$

$$
\mathrm{v}=\varrho_{1} \frac{\sin \beta}{\sin \gamma} .
$$

Aus $\triangle G_{1}$ ML ergiebt sich

$$
\begin{aligned}
G_{1} M: L M & =\sin \lambda: \sin x \\
G_{1} M & =G_{1} M_{1}-M_{1} \\
G_{1} M_{1} & =v \\
M M_{1} & =A M_{1}-A M \\
& =A E+Q_{1}-A M
\end{aligned}
$$

setzen wir $A E_{1}$ die Tiefe der vorderen Kammer, $=\xi$ und $\mathrm{AM}=\mathrm{LM}=\rho$ als Radius der Scleralkrümmung, so ergiebt sich aus obiger Gleichung schliesslich

$$
\sin \lambda=\frac{\left(v+g-\left(\xi+\rho_{1}\right)\right) \sin x}{\rho} .
$$

Aus dem rechtwinkligen Dreieck $M B N$ ergiebt sich

setzen wir $\Varangle \mathrm{BMN}=\tau$

$$
\text { tang. } \mathrm{BMN}=\frac{\mathrm{BN}}{\mathrm{BM}}
$$

$\mathrm{BN}=\mathrm{b}_{2}$, als die halbe Hornhautbasis

$$
\mathrm{AB}=\mathrm{e} \text {, als Hornhauthöhe, }
$$

so ist

$\mathrm{B} \mathrm{M}=\varrho-\mathrm{e}$ und

$$
\operatorname{tang} \tau=\frac{\mathrm{b}_{2}}{\rho-\mathrm{e}} .
$$

In $\triangle \mathrm{NML}$ ist

$$
\begin{gathered}
\overline{\mathrm{NL}}^{2}=\overline{\mathrm{NM}}^{2}+\overline{\mathrm{ML}}^{2}-2 \mathrm{NM} \cdot \mathrm{ML} \cos \mathrm{NML} \\
\Varangle \mathrm{NML}=\Varangle \mathrm{G}_{1} \mathrm{ML}-\Varangle \mathrm{G}_{1} \mathrm{MN} \\
=2 \mathrm{R}-(2+\varkappa+\tau) \\
\mathrm{NM}=\frac{\mathrm{h}_{2}}{\sin \tau}
\end{gathered}
$$

daher ist schliesslich

(7) $\mathrm{NL}=\sqrt{\frac{\mathrm{h}_{2}{ }^{2}}{\sin ^{2} \tau}+\varrho^{2}-\frac{2 \mathrm{~h}_{2} \varrho}{\sin \tau} \cos \left(180^{\circ}-(\lambda+x+\tau)\right)}$. 
Yon den in diesen 7 Formeln vorkommenden Grössen kamn man

$$
\Varangle \infty, \Varangle 0 \text { und } \mathrm{h}_{2}
$$

auf eine einfache Art und Weise messen, während die Grössen

$$
\varphi_{1}, \varrho \text {, e und } \xi
$$

einer directen genauell Messung nicht so leicht zugänglich sind. Ich habe sie daher nach den allgemeinen Durchschnittszahlen, welche ich zum Theil der „Karte des menschlichen Auges" von Flemming entnommen habe, folgendermaassen in Rechnung gesetzt:

Radius der vorderen Linsenfläche bei accommodationsloser Linse........... $\rho_{1}=10 \mathrm{~mm}$ Radius der Scleralkrümmung . . . . . . . $\varrho=12 \mathrm{~mm}$ Wirkliche Tiefe der vorderen Kammer ... . $\xi=3,7 \mathrm{~mm}$ Hornhauthöhe . . . . . . . . . . . . e $=2,5 \mathrm{~mm}$

Mit Hilfe dieser theils direct gemessenen, theils als Durchschnittszahlen angenommenen Grössen kann man aus den obigen 7 Formeln NL berechnen. Doch ist die Rechnung, obwohl sie sich in Folge des constanten Werthes verschiedener Grössen sehr vereinfacht, immerhin noch umständlich genug. Es ist daher vortheilhafter die graphische Methode zu wählen.

Zeichnet man nämlich den Horizontalschnitt eines Bulbus in zehnfacher Vergrösserung auf, und trägt auf der optischen Achse in der Entfernung $\mathrm{AB}=2,5 \mathrm{~mm}$ nach hinten von dem Hornhautscheitel die Senkrechte $\mathrm{BN}_{1}=$ der halben gemessenen Hornhautbasis $\left(h_{2}\right)$ nach beiden Seiten hin auf, so kann man die Linie $\mathrm{N}_{\mathbf{1}} \mathrm{G}$ sehr leicht construiren, indem man das Complement des $\Varangle \mathrm{AJN}_{1} \doteq \iota$ an $\mathrm{N}_{1} \mathrm{~B}$ in $\mathrm{N}_{1}$ anträgt, den $\Varangle \iota$ findet man nach den Formeln

$$
\begin{aligned}
\sin \beta_{1} & =\frac{1}{\mathrm{n}_{0}} \sin (\omega-0) \\
\text { und } \Varangle \iota & =0+\beta_{1} .
\end{aligned}
$$


Wo liegt die vordere Grenze des etc. Augenhintergrundes? 43 Auf der Linie $N_{1} G$ errichtet man dam eine Senkrechte $\mathrm{GM}_{1}=\varrho(10 \mathrm{~mm})$, deren freier Endpunkt auf AM liegt. An diese Linie trägt man in $\mathrm{G}$

$$
\Varangle \beta=76^{\circ} 36^{\prime} 22^{\prime \prime}
$$

an, der freie Schenkel treffe den mit $\mathrm{ML}=\mathrm{MA}=\varrho(12 \mathrm{~mm})$ um $\mathrm{M}$ beschriebenen Kreis in $\mathrm{L}$, so ist $\mathrm{NL}$ die gesuchte Grösse, welche man bei der angenommenen Vergrösserung von $1: 10 \mathrm{sehr}$ bequem noch bis auf $\frac{1}{10} \mathrm{~mm}$ genau ablesen kann. Da der Messungsfehler etwa $1 \mathrm{~mm}$ beträgt, so ist die Construction ausreichend genau.

Wir haben stets die Sehne NL und nicht den Bogen NL bestimmt, da es practisch entschieden einfacher und genauer ist, wenn man diese Sehne mit dem Zirkel resp. durch Visiren über einen Millimetermaasstab bestimmt, als wenn man durch ein gebogenes Papiermaass den Bogen misst, zumal derselbe gar kein Kreisbogen ist, da das Stück RL (Fig. 1) zwar ein Theil der Kugeloberfläche ist, welche mit ML um M beschrieben wird, $R N$ aber ganz anders gekrümmt ist, indem der Hornhautfalz etwas einspringt.

Es ist jetzt noch erforderlich, die Methode kennen zu lernen, durch welche man die in Betracht kommenden Grössen, insbesondere $\Varangle \omega$ und $\Varangle o$ bestimmen kann.

$\Varangle \omega$ ist der Winkel, welchen der Grenzstrahl mit der optischen Achse bildet. Um ihn zu messen, untersuchte ich zunächst, unter welchem Winkel zur Gesichtslinie man mit dem Augenspiegel noch rothes Licht vom Augengrunde erhält. $\mathrm{Zu}$ diesem Zwecke benutzte ich das Perimeter, da es mir darauf ankam, eine Methode anzuwenden, welche unter Umständen jeder Practiker ohne besonders kostspielige Apparate ausüben kann. Die Genauigkeit und leichte Ausführbarkeit der Messungen am Perimeter liess für die hier in Betracht kommenden Zwecke nichts zu wünschen übrig, so dass ich diese Methode für alle Winkelmessungen anwandte. 
An dem Förster'schen Perimeter liess ich die Kinnstütze durch einen untergelegten Holzklotz soweit erhöhen, dass das Auge des Untersuchten sich noch etwas höher befand als die durch den oberen Rand des horizontal gestellten Perimeterbogens gelegte Ebene. Für einmalige Messungen genügt es natürlich, die Stütze gexügend weit in die Höhe zu schrauben oder, falls dies nicht ausreicht, irgend einen Gegenstand von geeigneter Dicke zwischen Kinn und Stütze einzuschalten, um das Auge in die nöthige Höhe zu bringen. Als Fixationszeichen diente das sonst als Prüfungsobject benutzte weisse Quadrat, welches jedoch an dem verschiebbaren Schlitten nach oben statt wie gewöbnlich nach unten hin gerichtet war, was man am einfachsten mit etwas Klebwachs improvisiren kann. Der benutate Concavspiegel wurde an einem besonderen Schlitten befestigt, so dass er einerseits um seine verticale Achse drehbar war, andererseits aber noch höher und tiefer gestellt werden konnte. Dies lässt sich ziemlich einfach erreichen, wenn. man den Spiegel durch die vertical gestellte Bohrung einer horizontal stehenden Klemmschraube hindurchsteckt und durch Anziehen der Schraube fixirt. Es genügt auch vollständig, den Spiegel mit der Hand an einer bestimmten Stelle des Perimeterbogens so festzustellen, dass sein Stiel möglichst vertical steht.

Besondere Sorgfalt wurde bei allen Messungen darauf verwandt, dass das untersuchte Auge, die centrale Oeffnung des Concavspiegels, durch welche der Beabachter blickt, und das Fixationszeichen sowie die Flamme der dicht am Perimeter aufgestellten Lampe sich genau in einer Horizontalebene befanden.

Stellt man den Spiegel an einer bestimmten Stelle des Perimeterbogens fest und wirft mit ihm Licht in das beobachtete Auge, welches fest auf das Fixationszeichen gerichtet jeder Bewegung desselben folgt, so kann man es durch Verschieben des fixirten Objectes erreichen, dass die 
Wo liegt die vordere Grenze des etc. Augenhintergrundes?

schmale Sichel des von dem Angengrunde reflectirten rothen Lichtes, welche man bei stark seitlichem Hineinleuchten in das beobachtete Auge erblickt, gerade verschwindet. Die Entfernung des Spiegels von dem Fixationszeichen in Perimetergraden giebt dann den Neigungswinkel gegen die Gesichtslinie an, unter welchem der Augengrund eben noch sichtbar ist. Man kann sich hierbei leicht überzeugen, dass dieser Moment eintritt, sobald sich der undurchsichtige Hornhautrand mit dem gegenïberliegenden Pupillarrande scheinbar deckt. Doch ist es eigentlich nicht genau der Pupillarrand, sondern ein etwas mehr peripherer gelegener Theil der Iris, welcher in Frage kommt, wie wir bereits bei der theoretischen Betrachtung gesehen haben, was freilich bei der directen Beobachtung nicht zu entscheiden ist, da die Entfernung zwischen dem Pupillarrande und dem fraglichen Theile der Iris an und für sich schon sehr gering ist, bei dem schrägen Hineinblicken in das Auge aber noch kleiner wird, da sie in sehr erheblicher Verkürzung erscheint. Falls die Grenze der Sichtbarkeit des rothen Lichtes nach aussen hin bestimmt werden soll, wobei sich der Spiegel natürlich an der Nasenseite befinden muss, ist es nothwendig, den Kopf etwas nach der Seite des Spiegels hin zu drehen, damit der Nasenvorsprung nicht hinderlich ist.

Man erhält auf diese Weise den Winkel zwischen Gesichtslinie und Grenzstrahl, doch muss dieser Winkel erst auf den Winkel zwischen optischer Achse und Grenzstrahl zurückgeführt werden, da nur die optische Achse im Auge eine symmetrische Lage hat. Hierzu ist die Kenntniss des Winkels zwischen optischer Achse und Gesichtslinie $(\Varangle \gamma)$ erforderlich. $\mathrm{Zu}$ seiner Bestimmung benutzte ich eine $\mathrm{Me}$ thode, ähnlich der von Grossmann und Mayerhausen ${ }^{\mathbf{1}}$ ), da die ophthalmometrische Messung zwar genauer, aber

1) Archiv für Ophthalmologie, XXIII, 3, Seite 217. 
auch umständlicher ist. Das Princip dieser Methode ist Folgendes.

Befestigt man auf dem Bogen des Perimeters eine kleine Wachskerze, so entwirft dieselbe ein kleines, scharfes und genügend helles Spiegelbildchen auf der Hornhant, dessen Verbindungslinie mit der Wachskerze mit dem Krümmungsradius der Hornhaut an dem betreffenden Punkte nicht etwa mit dem radius vector der Ellipse - zusammenfällt, da ein Strahl, der nach der Reflexion zur Lichtquelle zurückkehrt, in der Richtung der betreffenden optischen Achse d. h. des Krümmungsradius verläuft.

Verschiebt man das Fixationszeichen am Perimeter so lange, bis das Hornhautbildchen, der Hornhautrand und die Lichtflamme sich in einer geraden Linie befinden, so giebt der Winkel zwischen Fixationszeichen und Flamme den Winkel zwischen dem Krümmungsradius am Hornhautrande und der Gesichtslinie an.

Benutzt man ein brennendes Stïckchen Wachsstock als leuchtendes Object, so kam man sehr gut durch den unteren blauen nicht leuchtenden Theil der Flamme hindurch Hornhautbildchen und limbus beobachten. Statt des Wachskerzchens kann man sich auch in derselben Weise wie vorher eines Concavspiegels eines schmalen Planspiegels bedienen, welcher von einer Lampenflamme ein virtuelles hinter dem Spiegel gelegenes Bild entwirft, das zur Erzeugung des Hornhautbildchens dient. Allerdings wird hier die Verbindungslinie des Spiegelbildes auf der Hornhaut mit dem zu seiner Erzeugung benutzten virtuellen Bilde der Lampenflamme in dem Planspiegel nicht immer genau mit der centralen Durchbohrung des Spiegels, durch welche der Beobachter hindurchblickt, zusammenfallen, indessen ist diese Abweichung, falls der Planspiegel nicht zu breit. ist, nur gering und fällt practisch wenig ins Gewicht, wie mir dies eine Anzahl vergleichender Messungen nach beiden Methoden gezeigt hat. 
Wo liegt die vordere Grenze des etc. Augenhintergrundes? 47

Auf diese Weise ist der Winkel zwischen Krümmungsradius am Hornhautrande und Gesichtslinie zu bestimmen. Denkt man sich nun aussen und innen den betreffenden Radius gezogen, so wird die Halbierungslinie des von beiden eingeschlossenen Winkels auf der Hornhautbasis senkrecht stehen. Diese Senkrechte fällt dann also mit der Hornhautachse und auch ungefähr mit der optischen Achse zusammen, und der Winkel zwischen dieser Halbirungslinie und der Gesichtslinie ist der gesuchte $\Varangle \gamma$. Nennt man den Winkel zwischen Hornhautradius am äusseren Cornealrande und Gesichtslinie $o_{1}$ und den entsprechenden Winkel innen $o_{2}$, so ist

$$
\Varangle \gamma=\frac{o_{1}-o_{2}}{2}
$$

wie leicht aus der geometrischen Betrachtung folgt.

Diese Methode, den $\Varangle \gamma$ am Perimeter zu bestimmen, dürfte sich vielleicht für manche Fälle, in denen es mehr auf eine schnell und leicht ausführbare als auf eine sehr genane Messung ankommt, empfehlen.

Mit Hilfe des $\Varangle \gamma$ lässt sich der gefundene Werth für den Winkel zwischen Grenzstrahl und Gesichtslinie auf die optische Achse reduciren. Wir erhalten so den $\subsetneq \omega$ in Fig. 1 und 2.

Für die Berechnung ist noch die Kenntniss des Durchmessers der Hornhautbasis erforderlich, welche man mittelst eines Millimetermaassstabes eventuell unter Zuhilfenahme einer Lupe (Pupillometer) messen kann. Die Tiefe der vorderen Kammer, der Krümmungsradius der vorderen und hinteren Linsenfläche und der Durchmesser des Bulbus ist. bedeutend schwieriger $z u$ finden und ohne besondere Instrumente überhaupt nicht zu messen, ich habe daher diese Grössen nach den allgemeinen Durchschnittszahlen angenommen, wie bereits oben erwähnt.

$\mathrm{Um}$ genaue Resultate $z \mathfrak{u}$ erhalten begnüigte ich mich in keinem Falle mit nur einer Messung, sondern machte 
bei jeder Untersuchung mindestens drei Ablesungen, aus welchen das Mittel gezogen wurde. Bei einiger Uebung stimmen die Resultate der einzelnen Messungen sebr gut mit einander überein, den möglichen Fehler schätze ich auf 20. Es wäre nicht besonders schwierig, die zu messenden Winkel noch genauer zu bestimmen, jedoch ist die Feststellung des Punktes, bei welchem das rothe Licht des Augengrundes noch eben sichtbar ist, nicht mit mathematischer Genauigkeit auszuführen, so dass eine allzugenaue Messung des betreffenden Winkels vollkommen überflüssig ist.

Auf Grund der gefundenen Werthe ist nach den angegebenen Methoden die Entfernung des periphersten, ophthalmoskopisch noch sichtbaren Punktes des Augenhintergrundes rom Hornhautrande zu berechnen, doch habe ich zur Prüfung der Richtigkeit der Rechnung diese Grösse auch direct $z u$ bestimmen versucht. Dies ist bei hell pigmentirten etwas prominenten Augen möglich. Lässt man nämlich, wie das Donders auch gethan hat, ein solches Auge stark nach aussen wenden, und dann das Licht einer aussen und hinten stehenden Lampenflamme auf die Hornhaut fallen, so erscheint nach innen von dem Hornhautrande auf der Selera ein umgekehrtes Bildchen der Flamme. Rïckt die Flamme immer weiter nach hinten, so versehwindet endlich das Bildchen, obwohl noch Licht auf die Hornhaut fällt. Es ist dies der Augenblick, in welchem durch die Pupille kein Licht mehr auf die Netzhaut gelangen kann. Bestimmt man am Perimeter, indem man die Flamme an einem festen Punkte aufstellt und das Fixationszeichen verschiebt, den Punkt, wo das Flammenbildchen gerade noch sichtbar ist, so kann man dessen Entfernung von dem Hornhautrande direct messen mit Hilfe eines Schiebezirkels mit Nonius und ebenso den Winkel oder richtiger Bogen zwischen Lampe und Fixationszeichen als Einfallswinkel des Grenzstrahles, welchen man natürlich erst auf die optische Achse reduciren muss. 
Wo liegt die vordere Grenze des etc. Augenhintergrundes? 49

Auf diese Weise ist eine directe Messung ausführbar, durch welche eine Controlle der berechneten Werthe möglich wird, nur ist dabei zu beachten, dass die Entfernung des Centrums des Scleralbildchens vom Hornhautrande nicht absolut genau zu messen ist, da das Flammenbildchen in Folge des durch die schräg gestellte also schlitzförmige Pupille einfallenden nur schmalen Lichtkegels sehr wenig hell ist und ferner der Hornhautrand bei der schlechten Beleuchtung - die Messung findet im völlig verdunkelten Zimmer statt, in dem nur die zur Erzeugung des Scleralbildchens benutzte Lampe brennt - nicht gerade als mathematisch genauer Punkt betrachtet werden kann. Auch stören sehr häufig die Lider die Messung etwas, so dass man sie erst weiter auseinanderziehen muss, ebenso zucken die Patienten, sobald man sich dem Auge mit dem Zirkel nähert nicht selten unwillkürlich zusammen, so dass das eben noch deutliche Scleralbild plötzlich verschwindet. Bei sehr prominenten Bulbis and besonders bei ausgebildetem Exophthalmus machen sich diese störenden Momente weniger geltend, so dass dann die Resultate der Messungen genauer werden, im Allgemeinen dürfte der Messungsfehler $1 \mathrm{~mm}$ kaum überschreiten.

Die Entfernung des Scleralbildchens vom Hornhautrande auf der äusseren Seite der Sclera ist nur bei stark hervorstehenden Augen zu messen, da für gewöhnlich der Nasenvorsprung den Lichteinfall von der inneren Seite des Auges her sehr beeinträchtigt.

Wir erhalten also einerseits die Grösse NL direct durch Messung, andererseits können wir sie aus den gemessenen Winkeln berechnen resp. construiren. Beide Werthe, der wirklich beobachtete und der theoretisch berechnete stimmen ziemlich gut mit einander überein, wenn man erwägt, dass der Messungsfehler allein etwa $1 \mathrm{~mm}$ beträgt, und dass die Tiefe der vorderen Kammer, der Durchmesser des Bulbus und der Krümmungsradius der vorderen Linsenfläche จ. Graefe's Archiv für Oplathalmologie. XXXV. 3. 
nicht genau bestimmt worden sind. Es muss die in der folgenden Tabelle vorkommende Differenz bis zu $1,3 \mathrm{~mm}$ also den Messungsfehlern zugeschoben werden.

Die Tabelle giebt eine Uebersicht über die von mir untersuchten Augen nach ihrer Refraction geordnet, indem an erster Stelle das Auge mit der hochgradigsten Myopie steht, dann schwächere Grade von Kurzsichtigkeit, weiterhin Emmetropie, Hypermetropie und schliesslich Aphakie folgen. Spalte 4 giebt die Refraction des betreffenden Auges nach der Zollscala, welche zunächst functionell mit Gläsern geprüft, dann aber auch meist noch mit Hilfe des Refractionsbestimmers ron Schmidt-Rimpler objectiv festgestellt wurde, zum Theil unter Anwendung von Atropin, worüber Colonne 6 genauere Auskunft giebt. Die scheinbare Weite der Pupille ist nur der Vollständigkeit halber in die Tabelle mit aufgenommen worden, da sie, wie wir bereits wissen, auf das Resultat kaum einen Einfluss ausübt.

Aus Colonne 8 ersieht man die bekannte Thatsache, dass $\Varangle \gamma$ bei Myopen kleiner ist als bei Emmetropen und Hypermetropen.

Spalte $9-12$ beziehen sich auf den Fall, dass das Licht von aussen einfällt, das Scleralbildchen resp. die fragliche Hintergrundsgrenze also innen liegt, während Colonne $13-16$ den umgekehrten Fall betreffen. Spaite 9 und 13 mit der Ueberschrift "Lampe“" bezeichnen die Stellung der Lampe, bei welcher das Scleralbildchen eben noch sichtbar ist, während ,Spiegel ${ }^{\star}$ über Colonne 10 und 14 sich auf die Lage des Augenspiegels bezieht, bei welcher noch rothes Licht rom Augengrunde zu erhalten ist. Die für den Augenspiegel gefundenen Werthe für $\Varangle \omega$ in Spalte 10 und 14 sind meist etwas grösser als die entsprechenden Grössen in der Spalte „Lampe“. Dieser Umstand findet seine Erklärung darin, dass man mit Hilfe des Augenspiegels zwar noch unter einem ziemlich grossen Einfallswinkel einen schmalen Streifen rothen Lichtes erkennen kann, dass 
aber, falls sich an Stelle des Spiegels eine Lampe befindet, der durch die schräg gestellte Pupille einfallende schmale Lichtkegel nicht mehr genügt, ein deutliches Scleralbildchen zu entwerfen. Es muss vielmebr durch Verkleinerung des Einfallswinkels das die Pupille passirende Lichtbüschel etwas verbreitert also lichtstärker gemacht werden, um noch ein deutliches Scleralbildchen zu erzeugen. Die Differenz zwischen Spalte 9 und 10 beträgt, ausser in Fall 11, stets nur wenige Grade und ist ausser durch die eben angeführten Verhältnisse zum Theil noch durch die sehr einfache, also nicht absolut genaue Methode der Winkelmessung bedingt.

Aus der Tabelle ergiebt sich für die gesuchte Grösse ein Werth von $7,6-9,8 \mathrm{~mm}$, im Durchschnitt $8,5 \mathrm{~mm}$. Die vorkommenden Schwankungen betragen also nur etwa $2 \mathrm{~mm}$.

Zufällig fand ich eine Beobachtung, welche diese Thatsache vollständig bestätigt. Laqueur berichtet nämlich im Centralblatt für Augenheilkunde 1888, Seite 291, von einer Scleralwunde, deren vorderes Ende $10 \mathrm{~mm}$ vom Hornhautrande entfernt lag, und welche als schwarzer Streifen, an dessen beiden Enden rundliche weisse Flecke lagen, mit dem Augenspiegel sichtbar war. Die vordere Grenze des sichtbaren Augengrundes lag in diesem Falle also dem Hornhautrande noch etwas näher als $10 \mathrm{~mm}$, was mit dem von mir gefundenen Durchschnittswerthe von $8,5 \mathrm{~mm}$ sehr gut übereinstimmt. Diese Beobachtung beweist zugleich, wie man sich übrigens leicht überzeugen kann, dass es möglich ist, von dem Augengrunde auch bis an die Grenzen seiner Sichtbarkeit ein deutliches Bild und nicht nur einen unbestimmten rothen Lichtreflex zu erhalten.

Da der Hauptfehler bei der theoretischen Berechnung entschieden durch die Annahme einer schematischen Linse gemacht worden ist, die directe Messung des Linsenradius aber ausserordentlich schwierig ist, so suchte ich einen geeigneten Fall von Aphakie, um an ihm dieselben Messungen anzustellen, da dann der Grenzstrahl nur durch die 


\begin{tabular}{|c|c|c|c|c|c|c|c|}
\hline 1. & 2. & 3. & 4. & 5. & 6. & 7. & 8. \\
\hline 栏 & Name und Aiter & 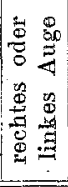 & $\begin{array}{c}\text { Re- } \\
\text { fraction }\end{array}$ & 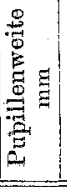 & $\begin{array}{l}\text { Mydriati- } \\
\text { cum }\end{array}$ & 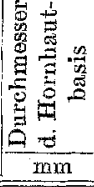 & $\not \gamma$ \\
\hline 1. & \multirow{2}{*}{ Adelheid R. $36 \mathrm{~J}$. } & r. & $\mathrm{M} \frac{1}{2^{1 / 4}}$ & 8,0 & $\begin{array}{l}\text { Homa- } \\
\text { tropin }\end{array}$ & 11,0 & $-2,7^{0}$ \\
\hline 2. & & 1. & $\mathrm{M} \frac{1}{2^{1} / 4}$ & 8,0 & $\begin{array}{l}\text { Homa- } \\
\text { tropin }\end{array}$ & 11,0 & $-2^{0}$ \\
\hline 3. & \multirow{2}{*}{ Emma T. $18 \mathrm{~J}}$. & $\mathrm{r}$. & $\mathrm{M} \frac{1}{4}$ & 7,2 & $\begin{array}{l}\text { Homa- } \\
\text { tropin }\end{array}$ & 10,6 & $+2^{0}$ \\
\hline 4. & & 1. & $\mathrm{M} \frac{1}{6}$ & 8,2 & $\begin{array}{l}\text { Homa- } \\
\text { tropin }\end{array}$ & 10,7 & $+1,9^{0}$ \\
\hline 5. & August W. $20 \mathrm{~J}$. & 1. & $\mathrm{M} \frac{1}{81 / 2}$ & 7,9 & Atropin & 11,3 & $+5,2^{0}$ \\
\hline 6. & \multirow{2}{*}{ Martha K. $29 \mathrm{~J}}$. & r. & N $\frac{1}{10}$ & 8,5 & Atropin & 12,0 & $+2,4^{0}$ \\
\hline 7. & & 1. & $\mathrm{M} \frac{1}{10}$ & 8,0 & Atropin & 12,0 & $+3^{0}$ \\
\hline 8. & Pauline J. $29 \mathrm{~J}$. & 1. & $M \frac{1}{30}$ & - & $\begin{array}{c}\text { ohne } \\
\text { Mydriat. }\end{array}$ & - & $+4,9^{\circ}$ \\
\hline 9. & \multirow{2}{*}{ Marie B. $\quad 31 \mathrm{~J}}$. & r. & $M \frac{1}{30}$ & 8,0 & Atropin & 12,0 & $+6^{0}$ \\
\hline 10. & & 1. & $\mathrm{M} \frac{1}{40}$ & 7,0 & $\begin{array}{l}\text { Homa- } \\
\text { tropin }\end{array}$ & 11,6 & $+5,3^{0}$ \\
\hline 11. & Willy M. $\quad 21 \mathrm{~J}$. & r。 & $M \frac{1}{40} ?$ & 8,8 & Atropin & 12,1 & $+5,4^{0}$ \\
\hline 12. & Paul St. & $\mathrm{I}$. & $\mathrm{E}$ & 8,0 & Atropin & 12,0 & $+7^{0}$ \\
\hline 13. & Christiane S. $42 \mathrm{~J}$. & 1. & $\mathrm{H} \frac{1}{60}$ & & & & \\
\hline 14. & Auguste B. $24 \mathrm{~J}$. & 1. & $\mathrm{H} \frac{1}{17}$ & - & Atropin & 一 & $+5,5^{\circ}$ \\
\hline 15. & Eduard B. $37 \mathrm{~J}$. & $r$ & $\begin{array}{l}\text { Apha- } \\
\text { kie }^{1} \text { ) }\end{array}$ & 7,0 & $\begin{array}{c}\text { ohne } \\
\text { Mydriat. }\end{array}$ & 11,4 & $+2^{0}$ \\
\hline
\end{tabular}

1) cf. Seite 54 Absatz 2 . 
Wo liegt die vordere Crenze des etc. Augenhintergrundes? 53

\begin{tabular}{|c|c|c|c|c|c|c|c|c|}
\hline 9. & 10. & 11. & 12. & 13. & 14. & 15. & 16. & 17. \\
\hline \multicolumn{2}{|c|}{$\begin{array}{c}\chi \omega \text { aussen } \\
\text { (zur } \\
\text { optischen Achse) }\end{array}$} & \multicolumn{2}{|c|}{ Grenze innen } & \multicolumn{2}{|c|}{$\begin{array}{c}\not{X} \omega \text { innen } \\
\text { (zur optischen } \\
\text { Achse) }\end{array}$} & Grenze aussen & aussen. & \multirow[t]{2}{*}{$\Varangle 0$} \\
\hline Lampe & |Spiegel & $\begin{array}{c}\text { messen } \\
\mathrm{mm}\end{array}$ & $\begin{array}{c}\text { rechnet } \\
\mathrm{mm}\end{array} \mid$ & Lampe & Spiegel & $\begin{array}{c}\text { messen } \\
\mathrm{mm}\end{array}$ & $\begin{array}{c}\text { rechnet } \\
\text { mm }\end{array}$ & \\
\hline $86,7^{\circ}$ & & 8,8 & 10,1 & $86^{\circ}$ & & 8,5 & 9,3 & $41,3^{0}$ \\
\hline $92^{\circ}$ & & 8,5 & 9,0 & $89^{\circ}$ & & 8,6 & 9,7 & $40,5^{0}$ \\
\hline $89,2^{0}$ & $93^{\circ}$ & 9,1 & 9,2 & $83^{\circ}$ & $83,2^{\circ}$ & & 11,2 & $39,3^{\circ}$ \\
\hline $90,9^{0}$ & $87,6^{\circ}$ & 9,2 & 9,6 & & $83,7^{\circ}$ & & 11,1 & $38,7^{\circ}$ \\
\hline $88,8^{\circ}$ & $91,5^{0}$ & 8,5 & 9,4 & & $92,2^{\circ}$ & & 9,4 & $41,0^{\circ}$ \\
\hline $97^{\circ}$ & $99,3^{0}$ & 7,8 & 7,4 & 92,4 & $90,4^{0}$ & 7,6 & 8,7 & $44,4^{0}$ \\
\hline $97,3^{\circ}$ & $97,3^{\circ}$ & 7,6 & 8,0 & 93,0 & $95,3^{\circ}$ & 8,8 & 8,7 & $42,0^{\circ}$ \\
\hline $89,1^{\circ}$ & $89,6^{\circ}$ & 8,1 & 9,2 & & $88,4^{0}$ & & 9,5 & $43,4^{\circ}$ \\
\hline $94,3^{\circ}$ & $97^{\circ}$ & 8,1 & 9,2 & & $96,3^{0}$ & & 9,6 & $35,5^{0}$ \\
\hline $95,6^{0}$ & $94,3^{\circ}$ & 8,5 & 9,0 & & $96,6^{\circ}$ & & 9,9 & $34,6^{0}$ \\
\hline $88,2^{\circ}$ & $97,4^{0}$ & 9,8 & 10,5 & & $90,1^{\circ}$ & & 10,0 & $36,4^{0}$ \\
\hline \multirow[t]{2}{*}{$88,5^{\circ}$} & $92,7^{0}$ & 9,25 & 9,25 & & $93,5^{0}$ & & 9,1 & $39,6^{\circ}$ \\
\hline & & 9,2 & & & & & & \\
\hline $98,8^{\circ}$ & $95,8^{0}$ & 7,7 & 8,3 & & $91,8^{0}$ & & 9,1 & $42,8^{0}$ \\
\hline $89,5^{\circ}$ & & 6,5 & 6,8 & & $94^{\circ}$ & & 9,4 & $40^{\circ}$ \\
\hline
\end{tabular}


Hornhaut gebrochen wird, deren optische Constanten leicht messbar sind.

Alle Fälle von Aphakie nach Kataraktoperationen erwiesen sich als unbrauchbar, da die fast ausnahmslos restirenden geringen Kapselreste jede genaue Messung unmöglich machten, doch führte mir der Zufall einen Patienten zu, bei welchem in Folge eines Traumas die vollständig klare Linse etwas nach aussen hin verschoben war (Fall 15 der Tabelle), so dass man an der Innenseite neben der Linse vorbei den Hintergrund erkennen konnte. Das Auge war daher in der inneren Pupillenbälfte als aphakisch anzusehen und das deutlich durchschimmernde Scleralbildchen machte eine genaue Messung möglich, während die Refraction in der äusseren Pupillenhälfte schwach hypermetropisch war.

Der gemessene Werth $6,5 \mathrm{~mm}$ stimmt mit dem berechneten von $6,8 \mathrm{~mm}$ gut überein. Der Grenzstrahl bildet in diesem Falle die Verbindungslinie des Hornhautrandes mit dem gegenüber liegenden Pupillarrande. Nimmt man an, die Linse befinde sich in dem Auge noch an ihrer alten Stelle, so würde der Grenzstrahl allerdings, da die Linse etwas über die Ebene der Iris bervorragt, mit der optischen Achse einen grösseren Winkel bilden als bei Aphakie, doch kann dieser geringe Unterschied vernachlässigt werden. Würde der Grenzstrahl durch die Linse gebrochen, so würde er einen 9,6 mm hinter dem Hornhautrande gelegenen Punkt des Augengrundes treffen, wie die Berechnung ergiebt. Bei der vorhandenen Aphakie beträgt die Entfernung aber nur $6.5 \mathrm{~mm}$, die ophthalmoskopische Grenze wird also durch die Linse um etwa $3 \mathrm{~mm}$ weit nach hinten hin verschoben.

Die Tabelle zeigt ferner noch, dass die Sichtbarkeit des Augengrundes, wie das bei dem ziemlich symmetrischen Bau des Auges nicht anders zu erwarten ist, nach aussen und innen sich fast gleich weit erstreckt. Allerdings ist die Entfernung nach aussen hin meist etwas grösser, die 
Wo liegt die vordere Grenze des etc. Augenhintergrundes? 55

Grenze des Augenhintergrundes also weiter nach hinten hin gerïckt, aber dies ist doch nicht immer der Fall, und vor allen Dingen sind directe Messungen aussen nur selten auszuführen, so dass die Controlle für die Richtigkeit der Beobachtungen fehlt. Auch für die nur schwer zu messende Grenze nach oben und unten werden wir ähnliche Werthe voraussetzen können, zumal sich die überhaupt vorkommenden Schwankungen innerhalb der sehr engen Grenze von $2 \mathrm{~mm}$ halten.

Was den Einfluss der verschiedenen Theile des Auges auf die Lage der ophthalmoskopischen Grenze betrifft, so ist schon erwähnt worden, dass die Tiefe der vorderen Kammer der wichtigste Factor ist. Daher erscheint bei Hypermetropie, bei welcher die vordere Kammer meist enger ist als bei Myopie, die Grenze etwas nach vorn hin gerückt, während sie bei Myopie nach hinten hin zurück weicht. Doch gilt dieses Gesetz nur im Allgemeinen, aber durchaus nicht für jeden einzelnen Fall.

Bei dem einzigen Falle von stärkerer Hypermetropie in der vorliegenden Tabelle (No. 14) beträgt die Entfernung $7,7 \mathrm{~mm}$, bei den höheren Graden von Myopie (M $\frac{1}{6}$ und mehr) schwankt sie von 8,5 bis $9,2 \mathrm{~mm}$ und beträgt durchschnittlich $8,9 \mathrm{~mm}$, während sie in den zwischenliegenden Fällen ungefähr in der Mitte steht zwischen 7,6 und 9,25 mm, im Durchschnitt $8,4 \mathrm{~mm}$. Fall 11 ist hinsichtlich seiner Refraction zweifelhaft, indem es sich um einen nicht genau zu bestimmenden Astigmatismus handelt.

Dievordere Grenze des ophthalmoskopisch sichtbaren Augengrundes liegt also 8,5 mm hinter dem Hornhautrande, bei Myopen etwas weiter, bei Hypermetropen etwas weniger weit, wobei der mögliche Fehler kaum mehr als $1 \mathrm{~mm}$ beträgt, bei Aphakie reducirt sich diese Entfernung auf $6,5 \mathrm{~mm}$. Dieses Resultat dürfte für alle practischen Bedürfnisse vollkommen genügen. 
Auf Grund der bisherigen Betrachtung können wir die Ausdehnung desjenigen Theiles des Augengrundes berechnen, welchen wir mit dem Augenspiegel übersehen können. Diese Grösse beträgt für den horizontalen Meridian $45 \mathrm{~mm}$ d. h. der sichtbare Theil der Netzhaut erstreckt sich ron dem hinteren Augenpol nach jeder Seite hin 22,5 mm oder 16 Papillenbreiten weit, wenn wir den Durchmesser der Sehnervenpapille $=1,4 \mathrm{~mm}$ setzen. Da sich dieser Werth in den übrigen Meridianen des Bulbus nur wenig ändert, so können wir ein Stück des Augengrundes übersehen, welches $770 \mathrm{mal}$ oder in runder Summe $800 \mathrm{mal}$ so gross ist wie die Papilla optica.

Zum Schlusse meiner Arbeit ist mir eine angenehme Pflicht, Hern Geheimrath Förster für die Anregung zu derselben, sowie für seine rege Antheilnahme und Unterstützung bei meinen Versuchen meinen ergebensten Dank auszusprechen. 